\title{
Recurrent and persistent pityriasis rosea: an atypical case presentation
}

Sai Yee $\underline{\mathrm{Chuah}}^{1}$, MBChB, MRCP, Hui Yi $\underline{\mathrm{Chia}}^{1}$, MBBS, MRCP, Hiok Hee $\underline{\operatorname{Tan}}^{1}$, MBBS, MRCP

\begin{abstract}
We report a case of atypical pityriasis rosea in a 24-year-old Malay man. He presented with an 11-month history of three recurrent and persistent episodes of pityriasis rosea associated with oral ulcers. The first episode lasted for one month and recurred within 14 days. The second episode lasted for three months and recurred within nine days. The third episode lasted for seven months. Although all three episodes were not preceded by any prodromal symptoms, a herald patch was noted on three different sites (the left iliac fossa, abdomen and chest) on each successive episode. Recurrent pityriasis rosea and its association with oral ulcers, although quite uncommon, have been reported in the literature. However, reports of multiple recurrences, with prolonged duration of each episode and very short remissions in between, have not been made. To the best of our knowledge, this is the first report of such unique presentation.
\end{abstract}

Keywords: atypical, oral ulcers, persistent, pityriasis rosea, recurrent

\section{INTRODUCTION}

Pityriasis rosea, while recognised as early as 1798 by Robert Willan, was first described by French physician Camille Melchior Gilbert only in 1860.(1) It is a common, acute, selflimiting papulosquamous eruption that is characterised by oval erythematous squamous lesions of the trunk and limbs, usually sparing the face, scalp, palms and soles. ${ }^{(1)}$ Typically, the disease begins with a solitary patch termed a 'herald patch', after which generalised eruption appears along Langer's lines of cleavage. The herald patch has been observed in over $50 \%$ of patients, and multiple herald patches have also been reported. ${ }^{(2)}$ Up to $69 \%$ of patients have a prodromal illness before the herald patch appears, suggestive of an infectious origin, although this remains unproven. ${ }^{(2,3)}$

\section{CASE REPORT}

A 24-year-old Malay man, who works as a full-time tutor, presented with an 11-month history of recurrent and persistent papulosquamous rashes. He first presented in December 2010 with a sudden onset of mildly pruritic eruptions on his trunk and thighs, which had persisted for a week. A larger oval patch appeared on his left iliac fossa a week before the other eruptions, which were also associated with multiple oral ulcers. There was no prodromal illness, the patient's medical history was unremarkable and he was not on any medication.

Physical examination revealed a papulosquamous rash on his face, trunk, upper limbs and thighs. A larger oval patch with a slightly elevated fine-scaling border (most likely the herald patch), was present on his left iliac fossa (Fig. 1). There was an aphthous ulcer on the left lateral border of the tongue. The

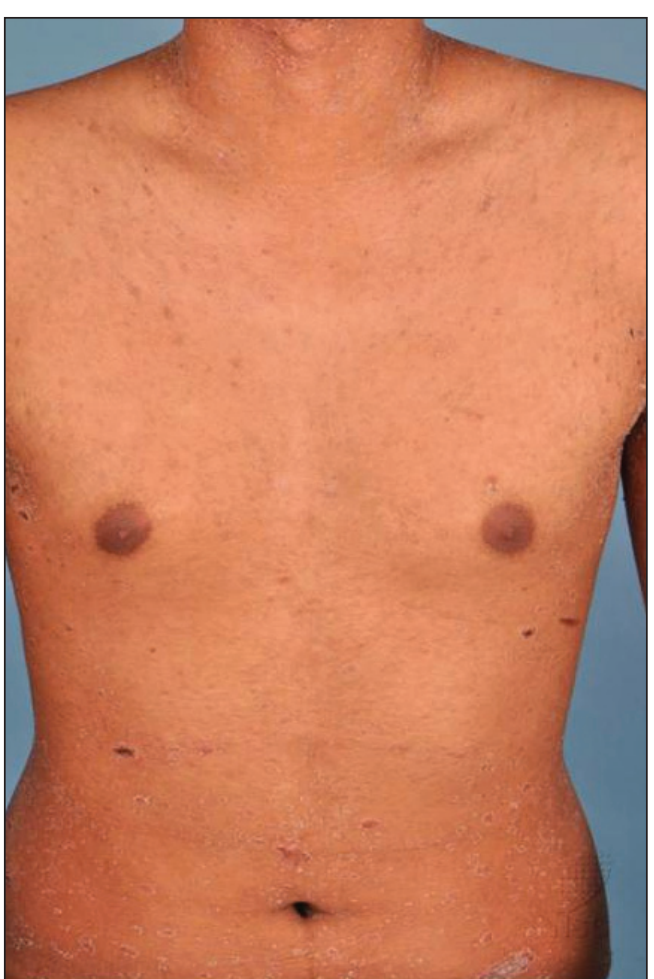

Fig. 1 Photograph shows scaly erythematous papules and plaques with collarette scaling on the trunk during the first episode. The large oval lesion on the left iliac fossa was thought to be a herald patch.

differential diagnoses included atypical pityriasis rosea, pityriasis lichenoides et varioliformis acuta, secondary syphilis and subacute cutaneous lupus erythematosus.

The patient's full blood count was normal. Rapid plasma reagin test, and screenings for human immunodeficiency virus and antinuclear antibodies were negative. Skin biopsy showed parakeratosis and orthokeratosis, with acanthotic epidermis

${ }^{1}$ Department of Dermatology, National Skin Centre, Singapore

Correspondence: Dr Sai Yee Chuah, Registrar, Department of Dermatology, National Skin Centre, 1 Mandalay Road, Singapore 308205. sychuah@nsc.gov.sg 


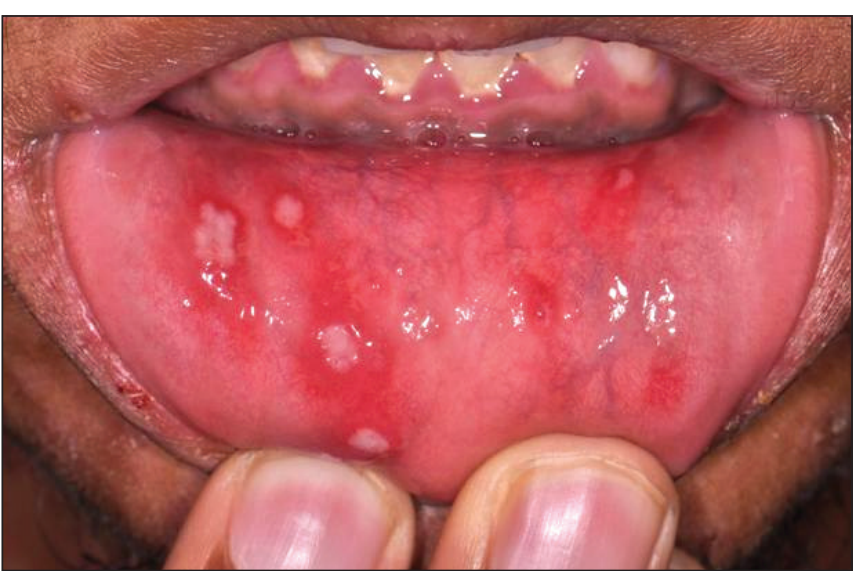

Fig. 2 Photograph shows the multiple aphthous ulcers on the lowe buccal mucosa. These ulcers were observed during the second episode.

associated with focal spongiosis. There was focal basal vacuolar alteration and lymphocytic exocytosis. The dermis showed the presence of melanophages and occasional red blood cells. Direct immunofluorescence was negative. Investigations suggested that the most likely diagnosis was atypical pityriasis rosea. We thus treated the patient with oral erythromycin $500 \mathrm{mg}$ twice daily for two weeks and topical betamethasone valerate $0.05 \%$ cream twice daily for the rash. The patient's rash subsided after a month.

Two weeks later, in January 2011, the patient developed a similar, mildly pruritic scaly rash over his trunk, upper limbs and thighs. In this episode, the herald patch had appeared on his mid-abdomen a few days before the smaller scaly eruptions, and his oral ulcers had recurred. However, as in the earlier episode, there was no prodromal illness or new medications. Examination revealed similar, but new, papulosquamous rash on his face, neck, trunk, upper limbs and thighs. A larger plaque with a peripheral collarette of scales was noted on the mid-abdomen. Multiple oral aphthous ulcers were also observed (Fig. 2). A diagnosis of recurrent atypical pityriasis rosea was made. The patient was prescribed with another course of oral erythromycin $500 \mathrm{mg}$ four times a day for two weeks, and topical betamethasone valerate $0.05 \%$ cream twice daily for the rash. However, a review two weeks later showed that his rash had not improved.

Repeat full blood count was normal. Herpes simplex virus isolation was done on the oral ulcers, but the results were negative. Repeat skin biopsy showed mild hyperkeratosis, with focal parakeratosis. There was focal spongiosis, with lymphocytic exocytosis. Some apoptotic keratinocytes were seen, with focal basal vacuolar alteration. A mild superficial perivascular infiltrate of lymphocytes was also observed (Fig. 3). The results of our investigations suggested atypical and recurrent pityriasis rosea in our patient. He was treated with acyclovir $400 \mathrm{mg}$ three times a day for a week and topical betamethasone valerate $0.05 \%$ cream. The rash and oral ulcers resolved after three months.

A third episode occurred nine days later in April 2011, with another new herald patch noted on the patient's chest a week

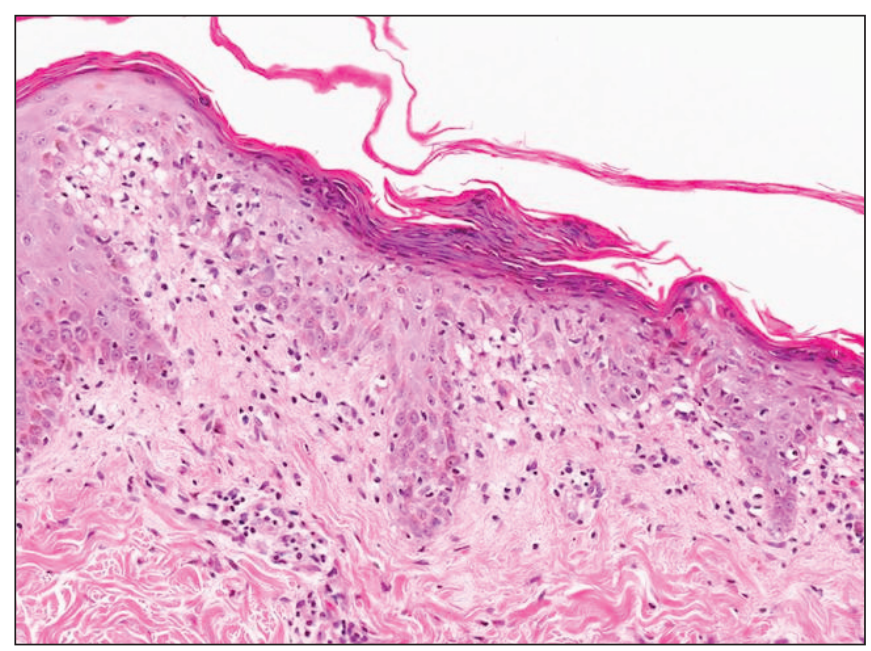

Fig. 3 Photomicrograph shows the histopathology of the biopsy specimen obtained during the second episode (Haematoxylin \& eosin, $\times 100)$. Hyperkeratosis with focal parakeratosis, focal spongiosis, and lymphocytic exocytosis are seen. A few apoptotic keratinocytes with focal basal vacuolar alteration, mild superficial perivascular infiltrate of lymphocytes, and extravasation of red blood cells are also seen.

before the papulosquamous eruptions on the axillae, neck and ears. There was also recurrence of the oral ulcers. As before, there was no prodromal illness or new medications. Medical examination revealed a papulosquamous rash with a collarette of scales on the patient's neck, ears, chest, axillae, upper limbs and thighs. There was a larger scaly plaque on his lower chest, which was likely the herald patch. Oral aphthous ulcers were also noted. The patient was prescribed another course of acyclovir $400 \mathrm{mg}$ three times a day for a week and topical mometasone furoate cream twice a day for the rash, with no improvement. He also tried homeopathy for two months with no effect. Narrowband ultraviolet B phototherapy was commenced three times a week for four months. The rash and oral ulcers subsided after seven months.

\section{DISCUSSION}

The overall incidence of pityriasis rosea is estimated to be 6.8 per 1,000 dermatological patients. ${ }^{(4,5)}$ Most episodes occur in patients aged 10-35 years. ${ }^{(1,2)}$ Pityriasis rosea shows a female predominance, with a male-to-female ratio of 1:1.5. ${ }^{(2)}$ However, in Singapore, Tay and Goh have reported a male preponderance, with a male-to-female ratio of 1.2:1. ${ }^{(5)}$

Typical eruptions of pityriasis rosea last 6-8 weeks, although durations as short as two weeks and as long as five months have also been reported. ${ }^{(1,2)}$ According to Björnberg and Hellgren, only $2.8 \%$ of patients relapse. ${ }^{(6)}$ However, Halkier-Sørensen reported a patient in whom relapses were observed annually for five consecutive years. ${ }^{(7)}$ Second attacks of pityriasis rosea are said to occur after an interval of a few months or many years. ${ }^{(8)}$ Drago et al, in their study of 430 patients, found that pityriasis rosea relapsed within 18 months in $3.7 \%$ of patients. ${ }^{(2)}$ However, the authors added that this relapse rate was probably an underestimation given that it was rare that the same doctor who made the original 
diagnosis would also be the doctor who observed the relapse in the patient. (2) This state of affairs may partly explain the lack of reports on multiple recurrences of pityriasis rosea in the literature.

In our patient, three relapses within an 11-month duration were observed. The interval between the relapses were very short, ranging from 9-14 days. The longest duration of the last relapse was seven months. To the best of our knowledge, such an atypical presentation of pityriasis rosea has not been reported in the literature. Furthermore, the association of oral ulcers with pityriasis rosea is uncommon. Oral ulcers have been reported to occur more often in dark-skinned people, ${ }^{(1,2)}$ and as our patient was of Malay ethnicity, this may have contributed to the atypical association of ulcers with pityriasis rosea that was observed in our study.

\section{REFERENCES}

1. Wood GS, Reizner G. Other papulosquamous disorders. In: Bolognia JL, Jorizzo JL, Rapini RP, eds. Dermatology. 2nd ed. Mosby: Elsevier Ltd, 2008:144-6.

2. Drago F, Broccolo F, Rebora A. Pityriasis rosea: an update with a critical appraisal of its possible herpes viral etiology. J Am Acad Dermatol 2009; 61:303-18.

3. Stulberg DL, Wolfrey J. Pityriasis rosea. Am Fam Physician. 2004; 69:87-91.

4. Chuh A, Zawar V, Lee A. Atypical presentations of pityriasis rosea: case presentations. J Eur Acad Dermatol Venereol 2005; 19:120-6.

5. Tay YK, Goh CL. One year review of pityriasis rosea at the National Skin Centre, Singapore. Ann Acad Med Singapore 1999; 28:829-31.

6. Björnberg A, Hellgren L. Pityriasis rosea: a statistical, clinical and laboratory investigation of 826 patients and matched healthy controls. Acta Derm Venereol Suppl (Stockh) 1962; 42 (Suppl 50):1-68.

7. Halkier-Sørensen L. Recurrent pityriasis rosea: new episodes every year for five years. A case report. Acta Derm Venereol 1990; 70:179-80.

8. Singh SK, Singh S, Pandey SS. Recurrent pityriasis rosea. Indian J Dermatol Venereol Leprol 1998; 64:237. 